\title{
Modeling of the Traveling Wave Antenna in view of the ICRF heating of DEMO
}

\author{
Andre Messiaen ${ }^{1, *}$ and Riccardo Ragona ${ }^{1,2}$ \\ ${ }^{1}$ Laboratory for Plasma Physics, Royal Military Academy, (LPP-ERM/KMS), BE-1000, Brussels, Belgium \\ ${ }^{2}$ Ghent University, Department of Applied Physics, 9000 Ghent, Belgium
}

\begin{abstract}
An upgraded version of the fast semi-analytical code ANTITER is used to model the refueled TWA sections of any arbitrary number of straps facing a low coupling plasma profile. The code computes the $\mathbf{Y}, \mathbf{Z}$ and $\mathbf{S}$ matrices of the antenna array from which the performances of the TWA sections and of their feeding system are deduced as a function of the geometry of the TWA. The model incorporates the feeding of the section by a resonant ring circuit recirculating the output power of each section. Approximate scaling laws guiding the design are derived for the antenna main parameters. A first optimization of the design for the DEMO antenna section is made and the resulting power capability of a DEMO system evaluated.
\end{abstract}

\section{Modeling of a TWA section}

\subsection{Introduction}

A Traveling Wave Antenna (TWA) system is presently considered for a reliable ICRF heating of DEMO in low coupling conditions [1]. The TWA is integrated in the first wall. It can be continuous all around the machine but has to be periodically refueled by power sources. We prefer to divide it in individually fed TWA sections to improve the reliability of the entire system and avoid large cross-coupling effects between the generators [2]. The paper studies the optimization of the TWA parameters and proposes a first conceptual electromagnetic design for DEMO.

\subsection{Basic relations and iterative impedance}

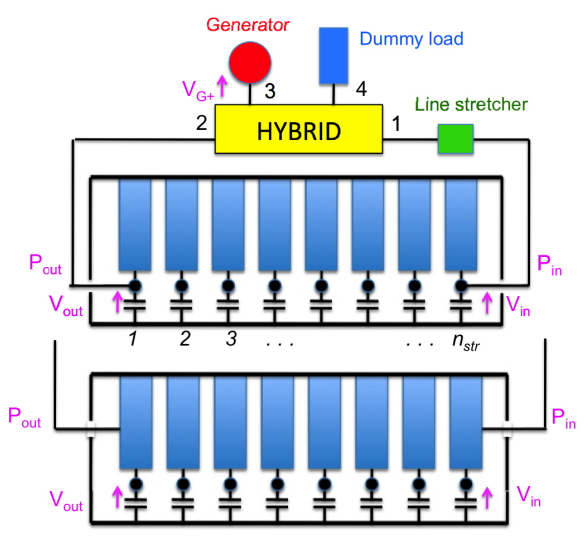

Fig. 1. TWA section with its feeding network (top without tap: $Z_{0}=Z_{\text {it }}$, bottom with tap: $Z_{0}=50 \Omega$ ).
Figure 1 shows a TWA section of $n_{\text {str }}$ straps and its feeding circuit. Each strap is tuned by its own capacitor. Only the first and last strap-capacitor connection points are connected to an external feeder. The admittance matrix $\mathbf{Y}_{\mathrm{oc}}$ seen towards the $n_{\text {str }}$ connection points is the sum of the strap admittance matrix $\mathbf{Y}_{\mathbf{S}}$ and the capacitor one $\mathbf{Y}_{\mathrm{C}}$ : i.e. $\mathbf{Y}_{\mathrm{oc}}=\mathbf{Y}_{\mathbf{S}}+\mathbf{Y}_{\mathrm{C}}$. The array impedance matrix $\mathbf{Z}_{\mathrm{a}}$, between the connection points to the two feeders, is immediately obtained from the terms of $\mathbf{Z}_{\mathrm{oc}}=\mathbf{Y}_{\mathrm{oc}}{ }^{-1}$ by the relation:

$$
\mathbf{Z}_{\mathrm{a}}=\left[\mathbf{Z}_{\mathrm{oc}}(1,1) \mathbf{Z}_{\mathrm{oc}}\left(1, n_{\mathrm{str}}\right) ; \mathbf{Z}_{\mathrm{oc}}\left(n_{\mathrm{str}}, 1\right) \mathbf{Z}_{\mathrm{oc}}\left(n_{\mathrm{str}}, n_{\mathrm{str}}\right)\right]
$$

The iterative impedance of the TWA section is such that $Z_{\text {in }}=Z_{\text {it }}$ if the section is terminated on the same impedance $Z_{\text {out }}=Z_{\text {it }}$. If the section is symmetric (i.e. $\mathbf{Z}_{\mathrm{oc}}(1,1)=$ $\mathbf{Z}_{\mathrm{oc}}\left(n_{\mathrm{str}}, n_{\mathrm{str}}\right)$ and $\left.\mathbf{Z}_{\mathrm{oc}}\left(1, n_{\mathrm{str}}\right)=\mathbf{Z}_{\mathrm{oc}}\left(n_{\mathrm{str}}, 1\right)\right)$ it is given by the relation:

$$
Z_{\mathrm{it}}=\left(\mathbf{Z}_{\mathrm{oc}}(1,1)^{2}-\mathbf{Z}_{\mathrm{oc}}\left(1, n_{\mathrm{str}}\right)^{2}\right)^{1 / 2}
$$

From $\mathbf{Z}_{\mathrm{a}}$, the corresponding scattering matrix $\mathbf{S}_{\mathrm{a}}$ is derived for a characteristic impedance $Z_{0}=Z_{\text {it }}$ or by approximation $Z_{0}=\operatorname{Re}\left(Z_{\mathrm{it}}\right)$. A tap on the first and last strap can be used for the connection to the feeders to bring $Z_{0}$ close to a standard $Z_{0}$ value (as $50 \Omega$ : see the bottom of figure 1). We recall that the TWA section behaves as a lossy transmission line section in its passband (see ref. [1]).

\subsection{Computation of the strap matrix $Z$}

ANTITER II [3] computes the field distribution by a Fourier integral expansion in the medium in front to the antenna box and an orthogonal function expansion inside 
the antenna box. The two expansions are matched at the box aperture to insure tangential fields continuity. First the contribution of each strap of the box to the field distribution is computed separately for a unit current flowing in the strap. As the field equations are linear the total field excited by the strap array is obtained by summing on the product of each contribution by its current complex amplitude. The active radiated power is obtained either by the flux of the Poynting's vector at the antenna aperture or by the e.m.f. method applied on the strap surface in the antenna box; The two computations are giving exactly the same result.

The impedance matrix $\mathbf{Z}=\mathbf{Y}^{-1}$ is computed by associating in pair the single strap contributions. The $(i, j)$ term of the $\mathbf{Z}$ matrix is obtained from the strap pair $(i, j)$ with even or odd excitation $\left(I_{\mathrm{i}}=+I_{\mathrm{j}}\right.$ or $\left.-I_{\mathrm{j}}\right)$. Applying the e.m.f. method to compute the corresponding complex power for the two excitations we have $2 \mathbf{P}_{\text {even }}(i, j)=\int\left(E_{\mathrm{i}}+\right.$ $\left.E_{\mathrm{j}}\right) .\left(J_{\mathrm{i}}+J_{\mathrm{j}}\right) * \mathrm{dS}$ and $2 \mathbf{P}_{\text {odd }}(i, j)=\int\left(E_{\mathrm{i}}-E_{\mathrm{j}}\right) .\left(J_{\mathrm{i}}-J_{\mathrm{j}}\right) * \mathrm{dS}$. For $\left|I_{\mathrm{i}}\right|=1 \mathrm{~A}$ we have also $2 \mathbf{P}_{\text {even }}(i, j)=\mathbf{R}_{\text {even }}(i, j)+\mathrm{i} \mathbf{X}_{\text {even }}(i, j)=$ $\mathbf{Z}_{\text {even }}(i, j)$ and $2 \mathbf{P}_{\text {odd }}(i, j)=\mathbf{R}_{\text {odd }}(i, j)+\mathrm{i} \mathbf{X}_{\text {odd }}(i, j)=\mathbf{Z}_{\text {odd }}(i, j)$.

Having computed the $\mathbf{Z}_{\text {even }}$ and $\mathbf{Z}_{\text {odd }}$ matrices then the impedance matrix $\mathbf{Z}=\mathbf{Y}^{-1}$ is obtained by the relation $\mathbf{Z}=$ $\left(\mathbf{Z}_{\text {even }}-\mathbf{Z}_{\text {odd }}\right) / 2$. The figure below shows the normalized values $\operatorname{Re}\left(\mathbf{Z}_{\mathrm{n}}(i, j)\right)$ and $\operatorname{Im}\left(\mathbf{Z}_{\mathrm{n}}(i, j)\right)$ of $\mathbf{Z}$ for each value of $i$ as a function of $j$ for an $n_{\text {str }}=8$ TWA section. The impedance $\mathbf{Z}_{\mathrm{n}}$ is expressed in $\Omega / \mathrm{m}$ and has to be multiplied by the strap length $l_{\text {str }}$ to find $\mathbf{Z}$ (for $l_{\text {str }}<<\lambda$ ).
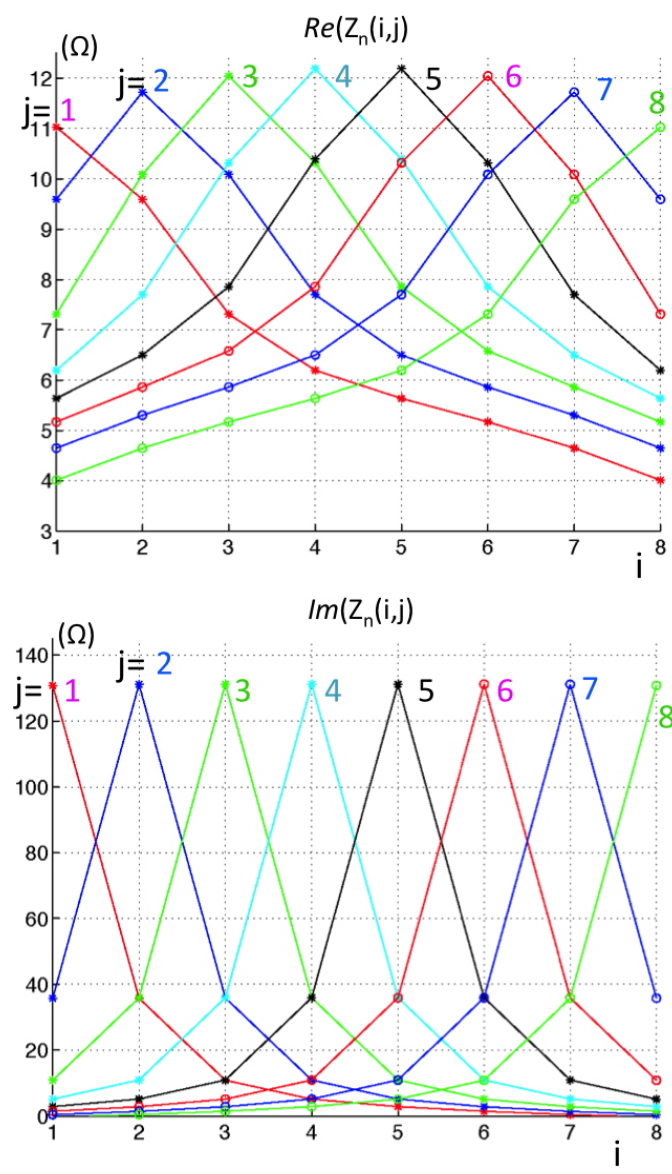

Fig. 2. Resistive and reactive parts of $\mathbf{Z}_{\mathbf{n}}$ versus strap number for the conditions given in section 2.1 and the reference plasma profile.

\subsection{Approximate relations and optimization of TWA}

Frequency band and iterative impedance. The frequency band is mainly determined by the choice of the tuning capacitance $C$ and the mean values of strap selfinductance $L=\operatorname{Im}\left(\left\langle\mathbf{Z}(i, i) / \omega_{0}\right\rangle\right)$ and mutual inductance to the adjacent straps $\left.M_{1}=\operatorname{Im}\left(<\mathbf{Z}(i, i \pm 1) / \omega_{0}\right\rangle\right)$. We take the same value of the capacitance $C=\left(L \omega_{0}^{2}\right)^{-1}$ for all the straps. Simple formulae are derived from the $n_{\text {str }}=2$ case that remain approximately valid for any strap number:

(i) The midband frequency of the TWA is $f_{0}=\omega_{0} / 2 \pi$ with $\omega_{\mathrm{o}}=(L C)^{-0.5}$ and the useful frequency band ranges between $f_{1}=(2 \pi)^{-1}\left(L+M_{1}\right)^{-1 / 2}$ and $f_{2}=(2 \pi)^{-1}\left(L-M_{1}\right)^{-1 / 2}$.

(ii) The iterative impedance is given by:

$$
Z_{\text {it }}=\omega_{0}\left(L / M_{1}\right)\left(L^{2}-M_{1}^{2}\right)^{0.5}
$$

A large frequency band and a low $Z_{\mathrm{it}}$ value requires a large ratio $M_{1} / L$ i.e. tightly magnetically coupled straps. Decay of strap voltage amplitude in the TWA section due to radiation. A TWA section can sustain traveling wave propagation in both directions [2]. We consider a traveling wave propagating in one direction from the input to the output of the TWA section. The radiated power by the strap $\mathrm{i}$ is given by: $P_{\text {str }, \mathrm{i}}=G_{\text {aeff }}\left|V_{\text {str }, \mathrm{i}}\right|^{2} / 2$ where $G_{\text {aeff }}$ is the array effective conductance. The incoming power along the TWA to the strap $i$ is given by: $P_{\mathrm{in,i}}=$ $\left|V_{\text {stri }}\right|^{2} /\left(2 Z_{\text {it }}\right)$ while the outgoing power from strap $i$ to the next strap by: $P_{\text {out }, \mathrm{i}}=P_{\mathrm{in}, \mathrm{i}+1}=P_{\mathrm{in}, \mathrm{i}}-P_{\mathrm{str}, \mathrm{i}}$. Therefore the radiated power decay along the TWA section can be computed as a function of the parameters $G_{\text {aeff }}$ and $Z_{\text {it }}$. We have $\left|V_{\mathrm{i}+1}\right|^{2}=\left|V_{\mathrm{i}}\right|^{2}\left(1-G_{\text {aeff }} Z_{\mathrm{it}} / 2\right)^{2}$ and the total radiated power by the section is given by the following geometric progression:

$$
P_{\mathrm{TWA}}=(1 / 2) \sum_{1}{ }^{N} G_{\text {aeff }}\left|V_{\text {in }}\right|^{2}\left(1-G_{\text {aeff }} Z_{\text {it }} / 2\right)^{2(N-1)}
$$

where $N=n_{\text {str }}$ This sum is equal to $P_{\text {TWA,max }}=\left(n_{\text {str }} / 2\right)$ $G_{\text {aeff }}\left|V_{\text {in }}\right|^{2}$ if all straps have same voltage amplitude $\left|V_{\text {str }, i}\right|=$ $\left|V_{\text {in }}\right|$. This relation shows that the product $G_{\text {aeff }} Z_{\text {it }}$ has to be minimized to avoid a too large decay of the radiated power along the TWA section. The output power of the section is given by:

$$
P_{\text {out }}=0.5 G_{\text {aeff }}\left|V_{\text {out }}\right|^{2}=0.5 G_{\text {aeff }}\left|V_{\text {in }}\right|^{2}\left(1-G_{\text {aeff }} Z_{\text {it }} / 2\right)^{2(N-1)}
$$

This is the power to be recirculated by the feeding system.

Resulting discussion for the optimization of the design Iof the TWA section. $G_{\text {aeff }}$ has to be maximized while $Z_{\text {it }}$ minimized in order to maximize the radiated power of a TWA section for a given value of $\left|V_{\text {in }}\right|$.

$G_{\text {aeff }}$ strongly depends on the plasma profile in front of the TWA. Moreover it also depends on the TWA geometry mainly due to its dependency on the selected $k_{/ \mathrm{MAX}}=\Delta \Phi / S_{\mathrm{z}}$ for which the radiated power spectrum $P_{\text {rad }}\left(k_{/ /}\right)$is maximum ([5], see also Fig. 4$) . \Delta \Phi$ is the mean phase difference between adjacent straps and it is directly related to the choice of the frequency in the passband of the TWA section. At midband frequency $f_{0}$ we have $\Delta \Phi \cong 75^{\circ}$. 
The $Z_{\text {it }}$ value determines the power $P_{\text {in }}$ fed to the TWA section for an input strap voltage amplitude $\left|V_{\text {in }}\right|$ and acts on the voltage decay along the TWA section by the product $Z_{\text {it }} G_{\text {aeff }} Z_{\text {it }}$ (see Eq.3) has to be chosen as low as possible to reduce the voltage amplitude and increase the efficiency of the TWA section. This means a high mutual coupling ratio $M_{1} / L$ and a small strap length $l_{\text {str. }}$. Low $L$ requires straps with large width $2 w_{z}$ and high $M_{1}$ requires small values of mid-strap inter-distance $S_{\mathrm{z}}\left(S_{\mathrm{z}}\right.$ and $w_{z}$ are defined in Fig. 3). A compromise has to be found between the contradictory request of small $S_{\mathrm{z}}$ with: (i) good voltage standoff between adjacent straps, (ii) large strap width to decrease $L$ and (iii) sufficiently small $\left|k_{/ \mathrm{MAX}}\right|=\Delta \Phi / S_{\mathrm{z}}$ value to maximize the coupling. A short strap length is also incompatible with good coupling. This is the reason why we propose, for increasing the effective strap length seen by the plasma, to cut a long strap in several grounded short straps having their currents in phase (see Fig. 6).

\section{Electromagnetic design for DEMO}

\subsection{Modeling conditions}

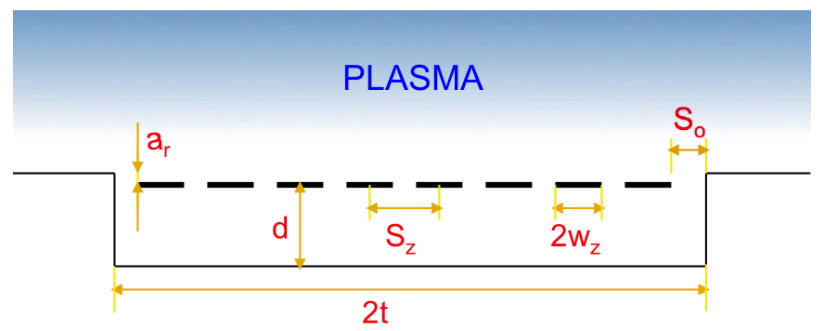

Fig. 3. Crossection of the TWA section in front of plasma.

Geometrical parameters. The geometrical parameters indicated on Figure 3 have been chosen as a compromise taking into account the preceding discussion $\left(a_{\mathrm{r}}=1.5 \mathrm{~cm}, d=20 \mathrm{~cm}, S_{\mathrm{z}}=25 \mathrm{~cm}, S_{\mathrm{o}}=42.5 \mathrm{~cm}, w_{\mathrm{z}}\right.$ $=10 \mathrm{~cm}$, strap length $l_{\mathrm{str}}=20 \mathrm{~cm}$, number of strap per section $n_{\text {str }}=8$ ).

Plasma profile. The profiles choosen are the ITER reference profile for low coupling condition "2010 Low" (considered as the most pessimistic one [4] (see profiles in [5] ) and the same profile shifted by 5 or $10 \mathrm{~cm}$ away from the antenna in order to decrease further the coupling. The distances antenna-LCMFS (last closed magnetic flux surface) are then respectively 23, 28 and $33 \mathrm{~cm}$. We choose a DT $\left(\mathrm{He}^{3}\right)$ plasma with $B_{\text {edge }} \approx 3.5 \mathrm{~T}$ at $f=50 \mathrm{MHz} . \mathrm{He}^{3}$ minority heating is one good single pass absorption scenario, characteristic that is assumed for the boundary condition in the plasma bulk.

Resonant ring feeding network. The system is shown in Fig. 1 and it is used to recirculate the output power of the TWA section. It has many advantages which are discussed in reference [1]. The modeling is made by connecting the $\mathbf{S}_{\mathrm{a}}$ matrix of the TWA section to the $4 \mathrm{X} 4$ matrix of the hybrid junction $\mathbf{S}_{\mathrm{HYB}}$. The characteristic impedance of all the system is taken equal to $Z_{\text {it }}$ (its value can be adjusted to e.g. $50 \Omega$ by a tap connection to the first and last straps as shown in Fig. 1). The complete network equations are solved for a given excitation by the generator. The line stretcher position is adjusted to obtain the ring resonance [1] (i.e. the phase difference of the traveling wave after one complete turn in the ring equal to $p 2 \pi$ with $p=1,2,3 \ldots)$.

\subsection{Main modeling results}

Figure 4 shows (i) the mean phase difference $\langle\Delta \Phi>$ of the current of adjacent straps versus frequency of the TWA section tuned at $50 \mathrm{MHz}$ and (ii) some corresponding radiated power $P_{\text {rad }}\left(k_{/ /}\right)$spectra in the bandpass for $42<f<56 \mathrm{MHz}$ and the reference plasma

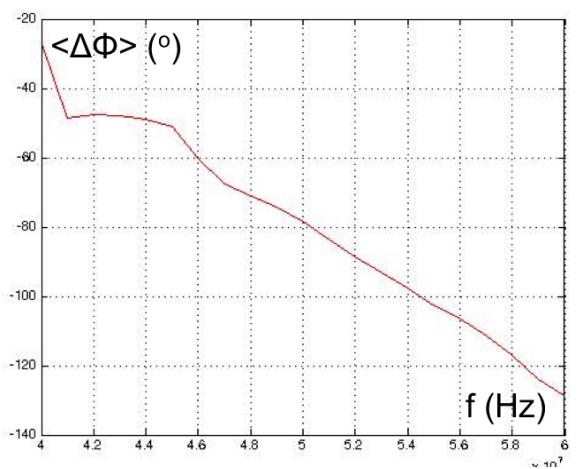

profile.

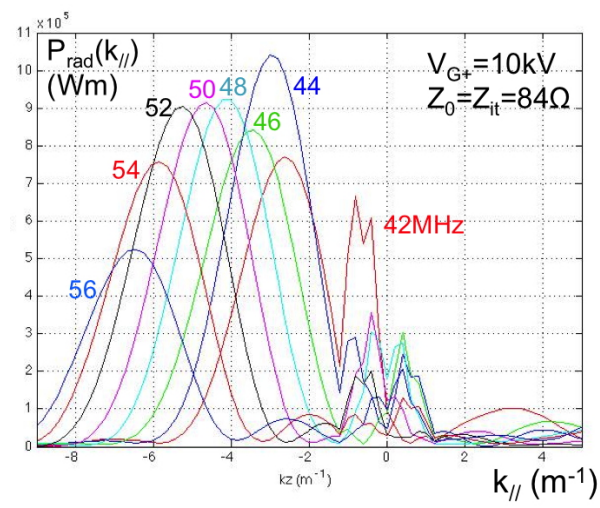

Fig. 4. $\left\langle\Delta \Phi>\right.$ and $P_{\text {rad }}\left(k_{/ /}\right)$versus frequency of the considered TWA section.

The most excited $k_{/ /}$is given by $k_{/ / \mathrm{MAX}}=\Delta \Phi / S_{\mathrm{z}}$ if the strap current amplitude $\left|I_{\text {str }}\right|$ and the inter-strap current phasing $\Delta \Phi$ are the same [5]. This relation is still valid for the TWA with decaying $\left|I_{\text {str }}\right|$ but with broader peak. When $f$ varies from 42 to $56 \mathrm{MHz}$ the $\left|k_{/ / \mathrm{MAX}}\right|$ value increases from 2.6 to $6.5 \mathrm{~m}^{-1}$. Note the larger excitation of coaxial modes (occurring for $\left|k_{/ /}\right|<k_{0}$ ) at low $\left|k_{/ / \mathrm{MAX}}\right|$. The decay of strap current and voltage amplitude along the TWA section is shown in Figure 5 at midband and for the reference and both removed plasma profiles.

The performances of the TWA section at midband are summarized in Table 1 for the 3 plasma profiles and for a maximum strap voltage amplitude of $15 \mathrm{kV}$. The TWA section couples to the plasma $930 \mathrm{~kW}$ but this value is reduced to $430 \mathrm{~kW}$ when the TWA distance to the plasma is increased by $10 \mathrm{~cm}$. Note that the interstrap voltage is larger and amount $18 \mathrm{kV}$. Indeed this voltage $\Delta V=\mid V_{\text {str }, \mathrm{i}}$ $V_{\text {str }, \text { i+1 }} \mid$ exceeds $\left|V_{\text {str }, \mathrm{l}}\right|$ when $60^{\circ}<\Delta \Phi<300^{\circ}$ if $\left|V_{\text {str }, \mathrm{i}}\right| \cong$ $\left|V_{\text {str }, i+1}\right|$ and at $50 \mathrm{MHz}$ we have $\Delta \Phi \approx 80^{\circ}$ 

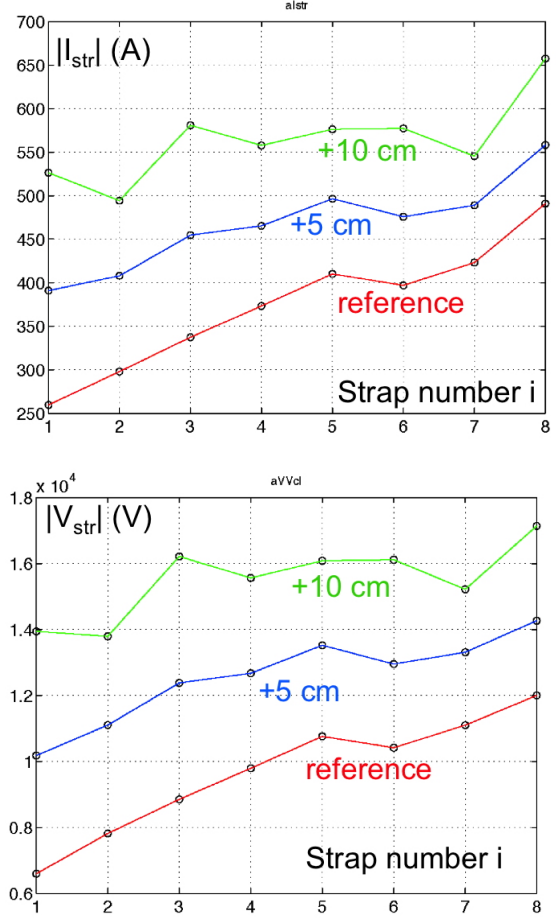

Fig. 5. Current and voltage amplitude of the straps for the three considered plasma profiles and a generator forward voltage $V_{\mathrm{G}+}=10 \mathrm{kV}$.

Table 1. Voltages and power of the TWA section (as defined in Fig. 1) for the three considered plasma profiles.

\begin{tabular}{|c|c|c|c|c|c|c|c|}
\hline $\begin{array}{c}\text { Antenna } \\
\text { plasma } \\
\text { distance }\end{array}$ & $\begin{array}{c}\boldsymbol{P}_{\mathrm{G}}=\boldsymbol{P}_{\text {rad }} \\
(\mathrm{MW})\end{array}$ & $\begin{array}{c}\boldsymbol{P}_{\text {in }} \\
(\mathrm{MW})\end{array}$ & $\begin{array}{c}\boldsymbol{P}_{\text {out }} \\
(\mathrm{MW})\end{array}$ & $\begin{array}{c}\boldsymbol{V}_{\text {in }} \\
(\mathrm{kV})\end{array}$ & $\begin{array}{c}\boldsymbol{V}_{\text {out }} \\
(\mathrm{kV})\end{array}$ & $\begin{array}{c}\boldsymbol{V}_{\mathrm{G}+} \\
(\mathrm{kV})\end{array}$ & $\begin{array}{c}\boldsymbol{V}_{\mathrm{G}+} \\
\mathbf{5 0 \Omega} \\
(\mathrm{kV})\end{array}$ \\
\hline$+10 \mathrm{~cm}$ & 0.428 & 1.18 & 0.75 & 15 & 12.2 & 8.75 & 6.58 \\
\hline$+5 \mathrm{~cm}$ & 0.636 & 1.25 & 0.62 & 15 & 10.7 & 10.52 & 7.98 \\
\hline reference & 0.930 & 1.30 & 0.37 & 15 & 8.24 & 12.5 & 9.65 \\
\hline
\end{tabular}

\subsection{First electromagnetic design for DEMO}

18 sections of quadruple TWA arrays are considered. Each section is constituted of two superposed and centrally grounded double tuned straps (see figure 6). The current are flowing in phase in each row of the four grounded straps: the effective strap length is then four times the one of the single strap $l_{\text {str }}$ considered in section 2.1. Therefore the total radiated power is $18 \times 4$ the one computed for the single array. The expected total power capability for a maximum strap voltage of $15 \mathrm{kV}$ is summarized in Table 2. We show for comparison (i) the larger expected radiated power with all individually fed straps and (ii) the power reduction due to vertical septa between the straps.

The main conclusions are:

- A set of 18 sections has a power capability of $67 \mathrm{MW}$ at $50 \mathrm{MHz}$ and exceeding $60 \mathrm{MW}$ in the frequency band $44-$ $52 \mathrm{MHz}$ for a maximum strap voltage of $15 \mathrm{kV}$ and a maximum inter-strap voltage of $18 \mathrm{kV}$ and for the reference low coupling plasma profile of ITER. The power capability of $67 \mathrm{MW}$ is reduced to 45 or $31 \mathrm{MW}$ if the distance antenna-plasma is increased by 5 or $10 \mathrm{~cm}$, i.e. distance antenna-LCMFS of 28 and $33 \mathrm{~cm}$.
Table 2. Total rf power coupled to DEMO by the proposed TWA system for the three considered plasma profiles.

\begin{tabular}{|c|c|c|c|}
\hline $\begin{array}{c}\text { Antenna plasma } \\
\text { distance }\end{array}$ & $\begin{array}{c}\text { Resonant ring } \\
\text { feed: } \\
\left|I_{\text {str }}\right| \text { decay }\end{array}$ & $\begin{array}{c}\text { Individual } \\
\text { strap feed: } \\
\text { no }\left|I_{\text {str }}\right| \text { decay }\end{array}$ & $\begin{array}{c}\text { Individual } \\
\text { strap feed: } \\
\text { septa between } \\
\text { straps }\end{array}$ \\
\hline$+10 \mathrm{~cm}$ & $30.8 \mathrm{MW}$ & $53.2 \mathrm{MW}$ & $27.7 \mathrm{MW}$ \\
\hline$+5 \mathrm{~cm}$ & $45.4 \mathrm{MW}$ & $77.5 \mathrm{MW}$ & $43.1 \mathrm{MW}$ \\
\hline reference & $66.4 \mathrm{MW}$ & $127.1 \mathrm{MW}$ & $73.3 \mathrm{MW}$ \\
\hline
\end{tabular}

- The peak of maximum radiation varies from $\left|k_{/ \mathrm{MAX}}\right|=$ 2.6 to $6.5 \mathrm{~m}^{-1}$ when changing the frequency from 42 to $56 \mathrm{MHz}$. For a maximum strap voltage of $15 \mathrm{kV}$ the radiated power exceeds $39 \mathrm{MW}$ in this frequency band and exceeds the value of 59MW from 42 to $52 \mathrm{MHz}$. This power capability could be further improved via edge gas puffing [6]. As a comparison, ANTITER predicts for the tight 24 straps array of the present ITER design facing the same reference plasma profile at $53 \mathrm{MHz}$ a power capability ranging from 9MW (for $\mathrm{k}_{/ \mathrm{MAX}}=7.5 \mathrm{~m}^{-1}$ ) to $15 \mathrm{MW}$ (for $\mathrm{k}_{/ / \mathrm{MAX}}=3.1 \mathrm{~m}^{-1}$ ) for a strap voltage amplitude of $41 \mathrm{kV}$.

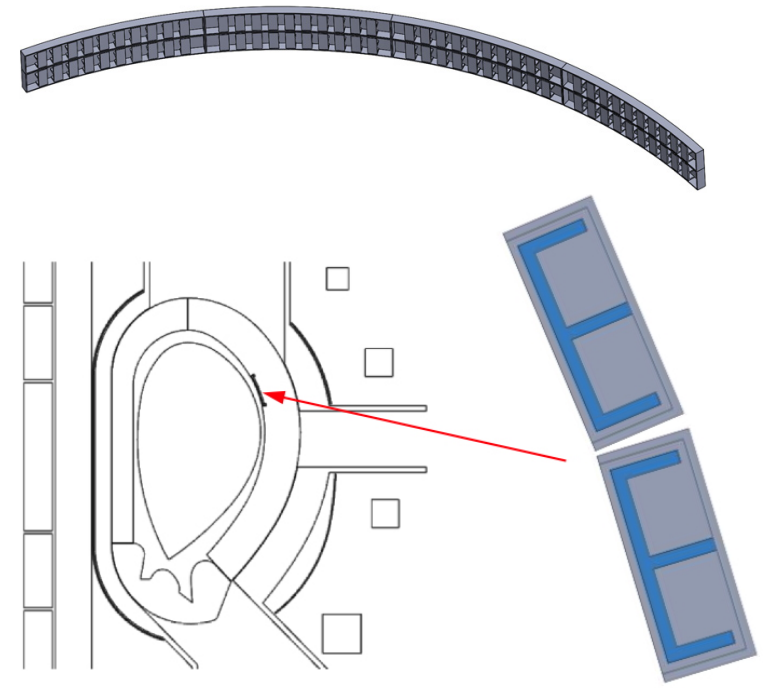

Fig. 6. Cross-section of the proposed TWA antenna system for DEMO. The sides of the centrally grounded double straps are the tuning capacitances. On top an overview of four segments.

This work has been carried out within the framework of the EUROfusion Consortium and has received funding from the Euratom research and training programme 2014-2018 under grant agreement No 633053. The views and opinions expressed herein do not necessarily reflect those of the European Commission.

\section{References}

1. R. Ragona and A. Messiaen, Nucl. Fusion 56 (2016) 076009.

2. R. Ragona and A. Messiaen, this conference.

3. A. Messiaen et al, Nucl. Fusion 50 (2010) 025026.

4. S. Carpentier and R. A. Pitts Report ITER_D_33y59M_v2,3 (April 2010).

5. A. Messiaen and R. Ragona, European Conference Abstracts (ECA) vol. 40A (2016), Leuven, Belgium.

6. P. Jacquet et al, Nucl. Fusion 56 (2016) 046001 